\title{
First-principles modeling of strain in perovskite ferroelectric thin films
}

\author{
OSWALDO DIÉGUEZ \\ Department of Materials Science and Engineering, \\ Massachusetts Institute of Technology, \\ 77 Massachusetts Avenue, Cambridge MA 02139, USA \\ DAVID VANDERBILT \\ Department of Physics and Astronomy, \\ Rutgers University, \\ 136 Frelinghuysen Rd, Piscataway NJ 08854, USA
}

November 8, 2018

\begin{abstract}
We review the role that first-principles calculations have played in understanding the effects of substrate-imposed misfit strain on epitaxially grown perovskite ferroelectric films. We do so by analyzing the case of $\mathrm{BaTiO}_{3}$, complementing our previous publications on this subject with unpublished data to help explain in detail how these calculations are done. We also review similar studies in the literature for other perovskite ferroelectric-film materials.
\end{abstract}

\section{Introduction}

Ferroelectrics materials are of technological importance due to their ability to sustain a macroscopic polarization that can be switched by the action of an electric field. In particular, the demand for smaller industrial components has drawn attention in the last few years to ferroelectrics in film form. These can sometimes be grown epitaxially in a coherent fashion with thicknesses beyond tens of nanometers. Part of what makes them interesting is that the properties of the thin films can differ significantly from those of the corresponding bulk form. Several factors contribute to these differences, e.g., the thickness of the film, the electrical boundary conditions imposed on it, the orientation of the substrate upon which the film grows, the presence of defects or stoichiometric variations, and the misfit strain imposed by the substrate on the film. The reader interested in the science of ferroelectric films can consult the recent reviews $[1,2,3,4,5,6,7,8$, especially the one by Rabe [6] that specifically addresses the theory of epitaxial strain effects.

In this paper we will be concerned with the role of the misfit strain in the structural behavior of ferroelectric thin films. In doing so we will restrict ourselves to perovskites, one of the most representative and studied families of ferroelectric materials. We will review how first-principles theories can be used to isolate the effect of epitaxial strain from the other film-related effects mentioned above. Section 2 introduces the structural phase diagrams that have proven to be very useful in understanding the properties of ferroelectric films. Section 3 explains in detail how first-principles calculations can be used to obtain those diagrams, focusing on the case of $\mathrm{BaTiO}_{3}$ as a prototypical example of a perovskite ferroelectric. This section is based in two of our previous publications 9 , 10, complemented by expanded results and detailed explanations that have not previously been published. In Sec. 4 we review similar studies that have been carried out for ferroelectric films composed of materials other than $\mathrm{BaTiO}_{3}$. Finally, we summarize briefly in Sec. 5 . 

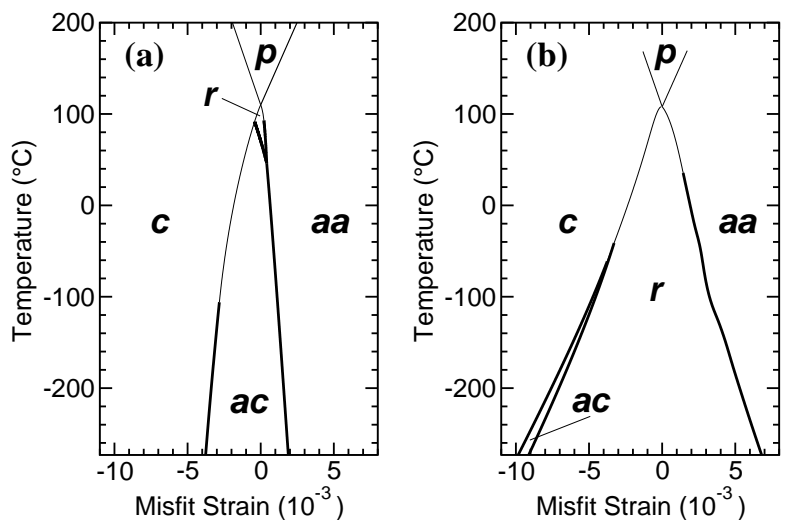

Figure 1: Phase diagrams of epitaxial $\mathrm{BaTiO}_{3}$ as predicted by the theory of Pertsev et al. [11]: (a) Using the parameters quoted in Ref. [11. (b) Using the parameters quoted in Ref. 12. First- and second-order phase transitions are represented by thick and thin lines, respectively. (Reprinted from Ref. [9]).

\section{Pertsev diagrams}

In a seminal paper, Pertsev, Zembilgotov and Tagantsev 11 introduced the concept of mapping the equilibrium structure of a ferroelectric perovskite material as a function of temperature and misfit strain, thus producing a "Pertsev phase diagram" of the observable epitaxial phases. The effect of epitaxial strain is isolated from other aspects of thin-film geometry by computing the structure of the bulk material with homogeneous strain tensor constrained to match a substrate having square surface symmetry and a given in-plane lattice constant. In addition, short-circuit electrical boundary conditions are imposed, equivalent to ideal electrodes above and beneath the film 11. Examples of such diagrams are shown in Fig. 1] the labeling of the phases therein is clarified in Table 1. Given the recognized importance of strain in determining the properties of thin-film ferroelectrics, Pertsev diagrams have proven to be of enormous value to experimentalists seeking to interpret the behaviour of epitaxial thin films and heterostructures.

In Ref. [1], the mapping was carried out using a phenomenological Landau-Devonshire model taken from the literature. Within this kind of theory, a thermodynamical potential describing the behaviour of the material is expanded in the relevant degrees of freedom. In the case of Ref. [11, the basic degrees of freedom were stress and polarization, with the temperature being incorporated via a linear temperature dependence of certain expansion parameters. For fixed misfit strain and temperature, a minimization over the three components of the polarization was carried out. Depending on the direction the polarization points at after the optimization, the film is in a distinctive phase labeled as indicated in Table 1.

This approach should give excellent results in the regime of temperature and strain in which the model parameters were fitted. However, it will generally be less accurate when extrapolated to other regimes. As an illustration of this, we pointed out in Ref. 9] that different sets of parameters can lead to significantly different diagrams even when exactly the same model is used, as shown in Fig. 1 Of course, such an empirical approach is also limited to materials for which all the needed experimental information is available.

Table 1: Summary of possible epitaxial $\mathrm{BaTiO}_{3}$ phases. In-plane cell vectors are fixed at $\mathbf{a}_{1}=a \hat{x}$, $\mathbf{a}_{2}=a \hat{y}$. Columns list, respectively: phase label; space group; out-of-plane lattice vector; number of free internal displacement coordinates; and form of the polarization vector.

\begin{tabular}{ccccc}
\hline Phase & $\mathrm{SG}$ & $\mathbf{a}_{3}$ & $N_{\mathrm{p}}$ & Polarization \\
\hline$p$ & $P 4 m m m$ & $c \hat{z}$ & 0 & 0 \\
$c$ & $P 4 m m$ & $c \hat{z}$ & 3 & $P_{z} \hat{z}$ \\
$a a$ & $A m m 2$ & $c \hat{z}$ & 4 & $P(\hat{x}+\hat{y})$ \\
$a$ & $P m m 2$ & $c \hat{z}$ & 4 & $P \hat{x}$ \\
$a c$ & $P m$ & $c_{\alpha} \hat{x}+c \hat{z}$ & 8 & $P \hat{x}+P_{z} \hat{z}$ \\
$r$ & $C m$ & $c_{\alpha}(\hat{x}+\hat{y})+c \hat{z}$ & 7 & $P(\hat{x}+\hat{y})+P_{z} \hat{z}$ \\
\hline
\end{tabular}




\section{First-principles calculations: the example of $\mathrm{BaTiO}_{3}$ films}

In this section we describe in detail how to apply a first-principles theory to the study of misfit strain effects in single-domain perovskite-oxide thin films grown on a substrate with square-lattice symmetry. In particular, we focus on the prototypical example of barium titanate. In Sec. 3.1 we show how this is done at a microscopic level, considering the physics of the individual electrons and ions in the film; this is what we call full first-principles calculations. In Sec. 3.2 we show how to apply a Landau-Devonshire theory in which first-principles calculations are used to find the coefficients in a Taylor expansion of the thermodynamical potential that describes the behaviour of the film; this is what we call first-principlesbased calculations. In both cases, the calculations are done for zero temperature. Finally, in Sec. 3.3 we show the results of applying a refined first-principles-based theory to take into account the effects of finite temperature.

\subsection{Full first-principles calculations}

\subsubsection{Theoretical details}

First-principles (i.e., ab initio) theories make use of the fundamental laws that govern the behaviour of electrons and nucleii to derive the macroscopical properties of a material. Among these, density-functional theory (DFT) [13 as implemented in the Kohn-Sham approach [14 is the most widely used to deal with systems such as those described here, since relatively large systems with many electrons can be treated accurately with a modest expenditure of computer time. Nowadays, several sophisticated computer codes capable of running such DFT calculations are available as open-source or inexpensive packages, including, e.g., ABINIT[15], the Quantum-ESPRESSO [16] suite of programs, Siesta[17, and VASP [18].

The first-principles DFT calculations described in this subsection were carried out using the VASP 18 . software package. The electron-ion interaction was described by the projector augmented-wave method [19; semicore electrons are included in the case of $\mathrm{Ba}\left(5 s^{2} 5 p^{6} 6 s^{2}\right)$ and $\mathrm{Ti}\left(3 s^{2} 3 p^{6} 4 s^{2} 3 d^{2}\right)$. The calculations employed the Ceperley-Alder [20] form of the local-density approximation (LDA) exchange-correlation functional; other flavours of this functional, like the generalized gradient aproximation, do not lead to substantial improvements in the case of $\mathrm{BaTiO}_{3}$ 21. We use a plane-wave basis set with a $700 \mathrm{eV}$ kinetic-energy cutoff and a $6 \times 6 \times 6$ Monkhorst-Pack 22] sampling of the Brillouin zone.

Readers wishing to become more familiar with the terminology used in the previous two paragraphs, or to know more about the conceptual framework behind DFT, can consult a recent review on the subject by Capelle 23]; see also references therein.

\subsubsection{Search for the most stable phases}

Given the constituents of the unit cell, DFT codes allow one to carry out a search for the structural configuration with the lowest energy. We did this for the case of $\mathrm{BaTiO}_{3}$ in Ref. 9] where, following Pertsev et al. [11, we worked with a bulk-like periodic system in which the film boundary conditions imposted by the misfit strain have been applied. We emphasize that there are no surfaces or interfaces in our calculation. Essentially, we take a single unit cell from the deep interior of the film and replicate it periodically in all three dimensions; this periodic structure is the one used for our calculations.

We performed optimizations of distorted configurations of the five-atom unit cell of the cubic perovskite structure in order to look for the six possible phases mentioned in Table1. Each starting structure had the $\mathrm{Ba}$ atoms fixed as determined by the substrate, and we displaced the $\mathrm{Ti}$ and $\mathrm{O}$ atoms along the direction of the polarization vector of each phase. Then, while retaining the symmetry determined by these displacements, we relaxed the atomic positions and the out-of-plane cell vector until the value of the Hellmann-Feynman forces and $z z, y z$ and $z x$ stress tensor components fell below $0.001 \mathrm{eV} / \AA$ and $0.005 \mathrm{eV}$, respectively. This process allowed us to determine the lowest-energy structure for each of the six possible phases.

Following this recipe for different values of the in-plane strain on the film, we obtained the energy curves of Fig. 2 (top panel). In some cases we started with a structure with a given symmetry, but found that the system relaxed to a structure with a higher symmetry; for example, starting with a structure having the symmetry of the $r$ phase at misfit strains exceeding $\sim 0.6 \%$, we find that it converges to a structure of $a a$ symmetry (collapse of the green curve onto the orange curve in the figure). It can be seen that the $c$ phase is the most stable structure for large negative (compressive) misfit strains, then the 
$r$ phase is most stable for an intermediate strain range, and finally the $a a$ phase is most stable at large positive (expansive) strains.

Because the phase transitions from $c \rightarrow r \rightarrow a a$ are both of second order, the curves join each other so smoothly that it is difficult to locate the precise phase boundaries from the energy curves alone. The boundaries can be located much more precisely by using a stability analysis. At each value of misfit in the $c$ phase, for example, we carry out finite-difference calculations of $x$ forces and the $x z$ stress as the atomic $x$ coordinates and the $x z$ strain are varied. The lowest eigenvalue of the resulting $6 \times 6$ Hessian matrix is plotted as the blue curve in the middle panel of Fig. 2, and its zero crossing identifies the critical misfit. A similar analysis is used to consider $z$ displacements and shear strains in the $a$ and $a a$ phases (red and orange lines respectively). By properly considering the zone-center phonons, the elastic shear, and linear cross-coupling between these two kinds of degrees of freedom, this analysis allows us to locate the second-order phase boundaries much more precisely than is possible through direct comparison of total energies. Since the $a$ phase is never the lowest-energy structure for any misfit strain (see top panel), we can ignore it, and we locate the $c \leftrightarrow r$ and $r \leftrightarrow a a$ transitions as indicated by the vertical dashed lines in the figure.

With first-principles calculations it is also possible to inspect the individual displacements of the atoms in each structure, as shown in the bottom panel of Fig.2. At the phase transition points, displacement patterns arise that characterize the phases on both sides of the transition.

The three panels in Fig. 2 give therefore a consistent picture showing how the structural properties of the film vary as a function of the misfit strain imposed on it. For large compressive strains, the lowest energy corresponds to the $c$ phase; on the other hand, for large tensile strains the $a a$ phase is favored. At a misfit strain of $s_{\max }(c)=-6.4 \times 10^{-3}(a=3.930 \AA)$, there is a second-order transition from the $c$ phase to the $r$ phase, with the polarization in the $r$ phase continuously rotating away from the $z$ direction as the misfit strain increases. At misfit strain $s_{\min }(a a)=6.5 \times 10^{-3}(a=3.981 \AA)$, the $r$ phase polarization completes its rotation into the $x y$ plane, resulting in another second-order transition to the $a a$ phase.

\subsubsection{Comments on the underlying assumptions}

Here we comment briefly on the effects of the assumptions made in the construction of this first-principles Pertsev diagram. In principle, we should consider the possibility of equilibrium structures with larger unit cells, particularly those with cell-doubling octahedral rotations (i.e., tilts). Such rotations have been shown to be important in $\mathrm{SrTiO}_{3}$, and could condense in $\mathrm{BaTiO}_{3}$ under sufficiently large misfit strains. As an example, we have checked that the paraelectric phase of the film is stable with respect to octahedral rotations about the [001] direction (with $\mathrm{M}_{3}$ symmetry) up to an epitaxial compressive strain

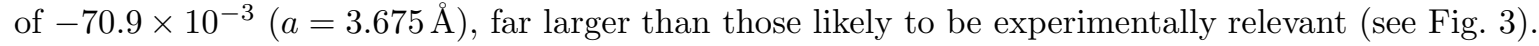

In addition, while we have studied only the effects of epitaxial strain, other physical effects may also be relevant to the structure and properties of thin films, such as atomic rearrangements at the filmsubstrate interface and free surface and the instability to the formation of multiple domain structures [24. Finally, our theory relies on the LDA to compute the exchange and correlation terms in DFT. This introduces small systematic errors in the calculation, the most important of which is probably the error in the equilibrium lattice constant. However, such errors are well understood and well characterized in perovskites, and tend to be similar for different materials of this class, so that there is a tendency for cancellation of errors when making relative comparisons of quantities such as misfit strains (see, for example, Ref. [25]).

It should be kept in mind, however, that for both films and superlattices, the assumptions that the system is in a single domain and that the epitaxial strain strongly dominates other factors will not be valid in all cases. Phase diagrams including multiple-domain states have, for example, been discussed in Refs. 26, 27, 24. Other influences that may be important include surface relaxation and reconstruction, atomic and electronic rearrangements at the interface, imperfectly compensated macroscopic electric fields, deviations from stoichiometry, and the presence of defects.

\subsection{First-principles-based calculations: a Landau-Devonshire model}

In a Landau-Devonshire approach, a ferroelectric system is described by a thermodynamical potential that is expanded as a Taylor series of the relevant degrees of freedom. For example, in the work of Pertsev and collaborators [11, the model is built by starting with the bulk free energy expanded in polarization 

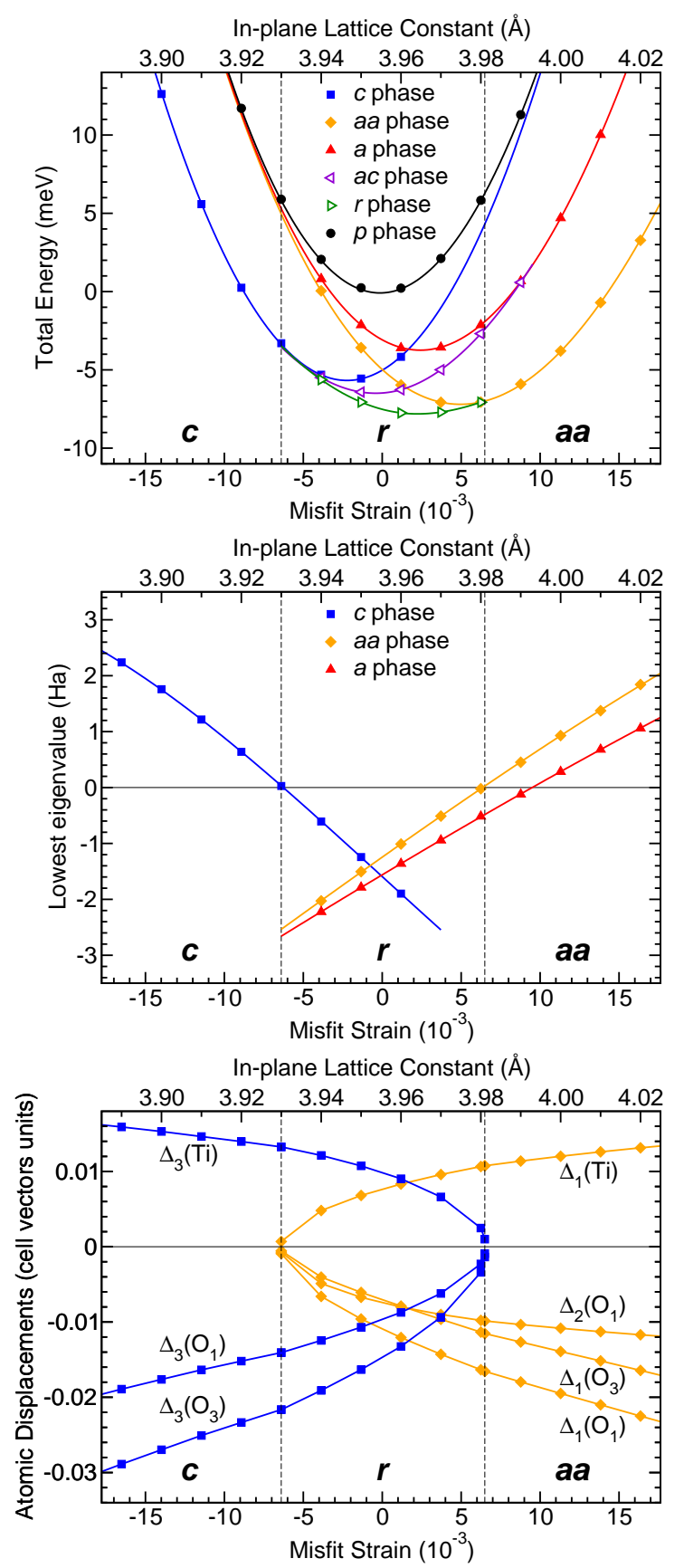

Figure 2: Top panel: energies of the possible epitaxial $\mathrm{BaTiO}_{3}$ phases for different misfit strains, as obtained from the full first-principles calculations. Center panel: value of the lowest non-trivial eigenvalue of the force-constant matrix for the three relevant phases; when it is zero, the second-order phase transitions denoted by vertical lines occur. Bottom panel: displacements of the atoms from the cubic perovskite cell positions, for the most energetically favorable configuration at a given misfit strain; $\Delta_{3}(\mathrm{Ti})$ labels the displacement of the Ti atom in units of the third lattice vector, etc. 


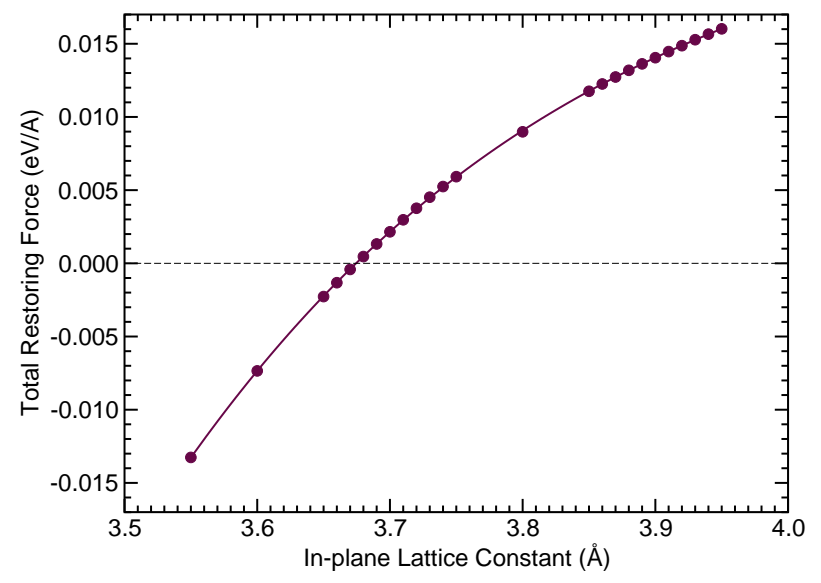

Figure 3: Value of the restoring force on the oxygen octahedra when they are subjected to a rotation about the [001] axis of $0.23^{\circ}$ in a pattern of $\mathrm{M}_{3}$ symmetry. A negative value indicates an instability leading to a doubled unit cell with oppositely rotated octahedra, but this only happens at a very high compressive strain (the equilibrium lattice constant is $3.96 \AA$ ).

and stress. The reference state is the paraelectric cubic perovskite phase at the bulk critical temperature $T_{\mathrm{c}}$, and the parameters are fit to reproduce experimental observations of the behavior near the bulk ferroelectric transition. For the dependence on epitaxial strain, a Legendre transformation is then made to obtain the potential as a function of polarization and misfit strain. Because of the way in which the parameters are fit, this Landau-Devonshire potential will give its most accurate results for small misfit strains and temperatures near the bulk $T_{\mathrm{c}}$.

It is also possible to develop models in the same spirit, but constructed from first-principles calculations. Compared with the phenomenological approach, such a first-principles-based theory has the advantage that the information needed for the model fit can readily be calculated in a consistent and accurate manner within the framework of the chosen first-principles approach. Then, once the fitting has been completed, such models can be used to compute detailed properties orders of magnitude faster than could be done using the full first-principles calculations. Thus, they can be applied to larger systems where the full calculations would be impractical. In this Section, we summarize how one such model [10. can be applied to study $\mathrm{BaTiO}_{3}$, providing additional details that were not included in the previous publication in order to clarify how the expansion coefficients were obtained.

\subsubsection{Formalism}

The starting point of this analysis is the parameterized total-energy expression presented by King-Smith and Vanderbilt in Ref. [25. This is a Taylor expansion around the cubic perovskite structure in terms of the six independent components $\eta_{i}$ of the strain tensor ( $i$ is a Voigt index, $\left.i=1-6\right)$ and the three Cartesian components $u_{\alpha}(\alpha=x, y, z)$ describing the amplitude of the soft mode defined by the pattern of eigen-displacements associated with the smallest eigenvalue of the (zone-center) force-constant matrix.

The energy (per unit cell) of the bulk material can be expressed as a sum

$$
E=E^{\text {elas }}\left(\left\{\eta_{i}\right\}\right)+E^{\text {soft }}\left(\left\{u_{\alpha}\right\}\right)+E^{\text {int }}\left(\left\{\eta_{i}\right\},\left\{u_{\alpha}\right\}\right),
$$

of a term $E^{\text {elas }}$ arising purely from strain, a term $E^{\text {soft }}$ arising purely from the soft-mode amplitude, and a term $E^{\text {int }}$ describing the interactions between these two kinds of degrees of freedom. The zero of energy is taken to correspond to the cubic reference structure. For crystals with cubic symmetry, the strain energy is given, to second order in strain, by

$$
E^{\text {elas }}\left(\left\{\eta_{i}\right\}\right)=\frac{1}{2} B_{11}\left(\eta_{1}^{2}+\eta_{2}^{2}+\eta_{3}^{2}\right)+B_{12}\left(\eta_{1} \eta_{2}+\eta_{2} \eta_{3}+\eta_{3} \eta_{1}\right)+\frac{1}{2} B_{44}\left(\eta_{4}^{2}+\eta_{5}^{2}+\eta_{6}^{2}\right),
$$

where $B_{11}, B_{12}$, and $B_{44}$ are related to the elastic constants of the crystal by factors of the cell volume. The soft-mode energy given in Ref. 25] contains terms up to fourth-order in the soft-mode amplitude,

$$
E^{\mathrm{soft}}\left(\left\{u_{\alpha}\right\}\right)=\kappa u^{2}+\alpha u^{4}+\gamma\left(u_{x}^{2} u_{y}^{2}+u_{y}^{2} u_{z}^{2}+u_{z}^{2} u_{x}^{2}\right),
$$


where $u^{2}=u_{x}^{2}+u_{y}^{2}+u_{z}^{2}, \kappa$ is twice the soft-mode eigenvalue, and $\alpha$ and $\gamma$ are the two independent symmetry-allowed fourth-order coefficients describing the cubic anisotropy. Finally, the interaction between the strains and the soft-mode amplitude is given to leading order by

$$
\begin{gathered}
E^{\mathrm{int}}\left(\left\{\eta_{i}\right\},\left\{u_{\alpha}\right\}\right)=\frac{1}{2} B_{1 x x}\left(\eta_{1} u_{x}^{2}+\eta_{2} u_{y}^{2}+\eta_{3} u_{z}^{2}\right)+\frac{1}{2} B_{1 y y}\left[\eta_{1}\left(u_{y}^{2}+u_{z}^{2}\right)+\eta_{2}\left(u_{z}^{2}+u_{x}^{2}\right)+\eta_{3}\left(u_{x}^{2}+u_{y}^{2}\right)\right] \\
+B_{4 y z}\left(\eta_{4} u_{y} u_{z}+\eta_{5} u_{z} u_{x}+\eta_{6} u_{x} u_{y}\right),
\end{gathered}
$$

where $B_{1 x x}, B_{1 y y}$, and $B_{4 y z}$ are the phonon-strain interaction coefficients. All the coefficients in these three parts of the total-energy expression can be obtained from first-principles calculations on a series of distorted structures as described in Refs. 25, 10. In Fig. 4 we present previously unpublished results of our first-principles calculations that were used as the basis for fitting these coefficients for the case of $\mathrm{BaTiO}_{3}$.

Once we have an expression for the energy of bulk $\mathrm{BaTiO}_{3}$, we can develop a potential appropriate for describing films. In the case of coherent epitaxy, where strain elements $\eta_{1}, \eta_{2}$ and $\eta_{6}$ are constrained while the others are free, this expression is

$$
\begin{gathered}
\tilde{G}=\left(A_{\bar{\eta} \bar{\eta}} \bar{\eta}^{2}+A_{\bar{\eta} \sigma} \bar{\eta} \sigma+A_{\sigma \sigma} \sigma^{2}\right)+\left(B_{\bar{\eta}} \bar{\eta}+B_{\sigma} \sigma+B\right) u_{x y}^{2}+\left(C_{\bar{\eta}} \bar{\eta}+C_{\sigma} \sigma+C\right) u_{z}^{2} \\
+D u_{x y}^{4}+E u_{z}^{4}+F u_{x y}^{2} u_{z}^{2}+H u_{x y}^{4} \sin ^{2} \theta \cos ^{2} \theta
\end{gathered}
$$

where $\sigma=\sigma_{z z}$ is a possible external uniaxial stress applied perpendicular to the film, $u_{x y}=\left(u_{x}^{2}+u_{y}^{2}\right)^{1 / 2}$, and $\theta=\tan ^{-1}\left(u_{x} / u_{y}\right)$, so that

$$
\begin{aligned}
& u_{x}=u_{x y} \cos \theta \\
& u_{y}=u_{x y} \sin \theta .
\end{aligned}
$$

The detailed expressions relating the coefficients appearing in Eq. (5) back to the KSV coefficients in Eqs. (244) are given in Ref. [10].

Once we have determined the set of coefficients in Eq. (5), we can predict the phase diagram as a function of misfit strain $\bar{\eta}$ and the normal external stress $\sigma$ by minimizing $\tilde{G}$ to find the values of the ground-state soft-mode amplitude components. For a fourth-order theory like the present KSV expression, the entire optimization process can be done analytically, since it is possible to compute first and second derivatives of $\tilde{G}$ and to do a stability analysis of the various possible phases.

\subsubsection{Testing the model: zero perpendicular external stress}

We first consider the usual case in which the external perpendicular stress $\sigma$ vanishes. Figure 5 (top) shows the energy curves of the various phases as predicted by the first-principles-based KSV theory (right panel) compared with the full DFT results (left panel). The agreement between the two sets of results is very good, with the small differences arising from two sources. First, the first-principles calculations in Ref. [9] (and in Sec. 3.1] were performed using the projector-augmented wave method, [19] while the firstprinciples calculations used to obtain the KSV coefficients referred here were performed using ultrasoft pseudopotentials [28]. Second, there are the intrinsic errors associated with the use of a Taylor expansion that has been truncated as described in the previous section; these errors are expected to grow as the strain and soft mode amplitudes increase. The bottom panels in Fig. 5 show the displacements of the atoms from their centrosymmetric perovskite positions as the strain is varied. Again, the agreement between the KSV results (right) and the full DFT results (left) is very good. In particular, the squareroot behavior predicted by the KSV theory is exhibited by the more exact DFT calculations.

\subsubsection{Other results}

In Ref. [10] we studied the effect of external stress $\sigma$ applied perpendicular to the film in addition to the effect of misfit strain. We did this for $\mathrm{BaTiO}_{3}$ and other seven perovskites, constructing a phase diagram for each in the two-dimensional space of normal stress and misfit strain. We found that the stress-strain phase diagrams obtained for all of the perovskites show a universal topology with straight-line phase boundaries meeting at a single crossing point. A detailed interpretation of the those results was given in Ref. [10, together with an analysis of the variables that drive each kind of behavior seen for each film. In the same article we also presented the computed polarization of the films and related these results to the concept of polarization matching. 
(a)

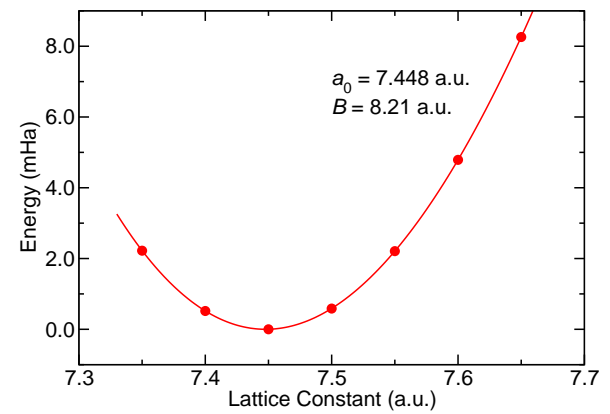

(b)

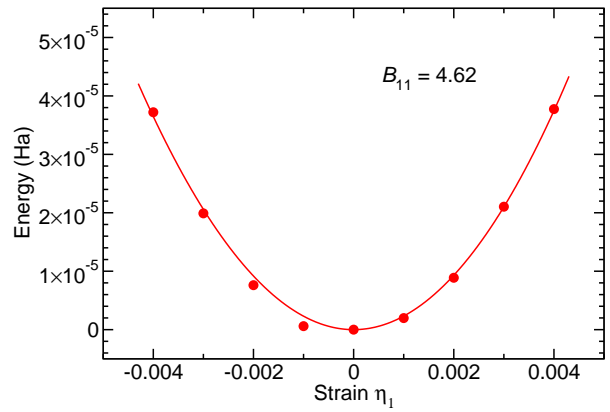

(d)

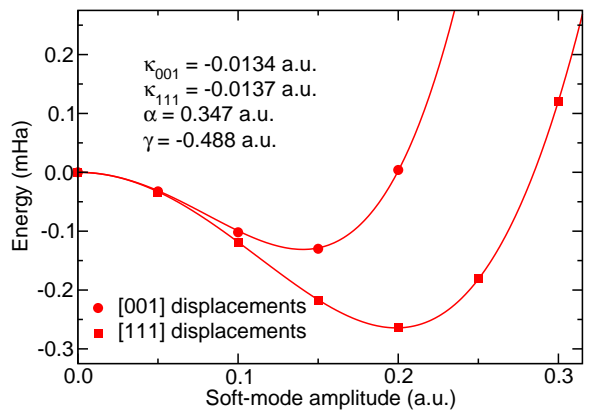

(f)

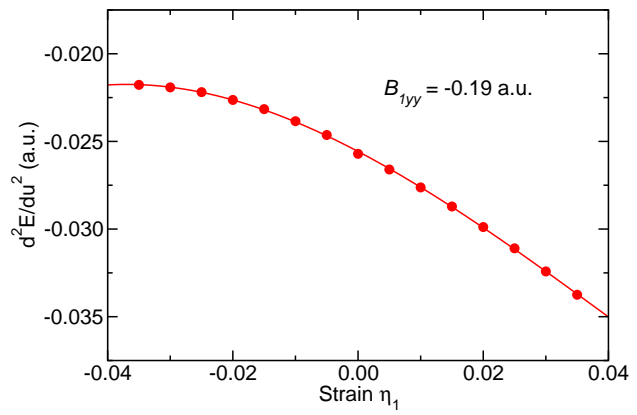

(c)

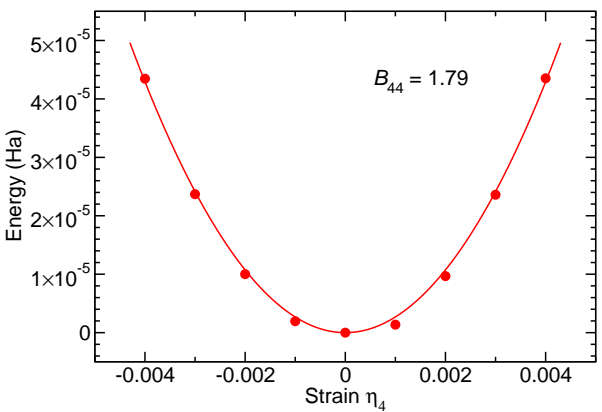

(e)

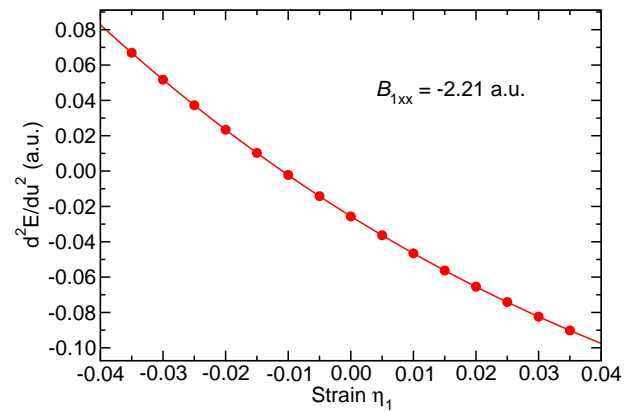

(g)

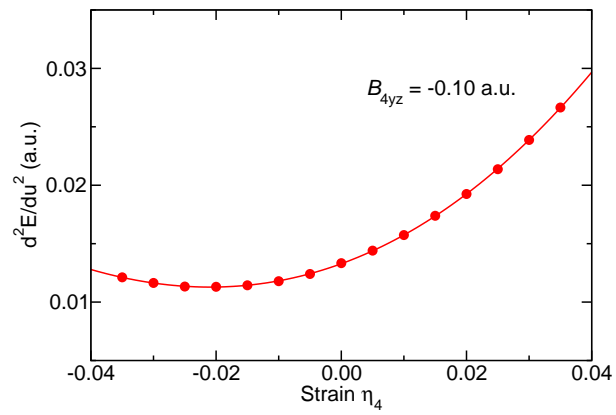

Figure 4: Results of first-principles calculations used to fit the coefficients of the first-principles-based model for bulk $\mathrm{BaTiO}_{3}$ as described in this section. In each panel, each symbol corresponds to a full first principles calculation, while each curve is a low-order polynomial fit to the data. The ground-state lattice constant and bulk modulus are obtained from the data in (a), through the use of the Birch equation. $B_{11}$ and $B_{44}$ are related to the curvature of the third-order polynomials of graphs (b) and (c); $B_{12}$ is obtained from the relation $B=B_{11}+2 B_{12}$. The coefficient $\kappa$ is half the lowest eigenvalue of the force constant matrix, obtained using finite differences and the ability of the first-principles programs to compute forces on the atoms. The coefficients $\alpha$ and $\gamma$ are obtained from the double-well sixth-order polynomial in (d). Finally, $B_{1 x x}, B_{1 y y}$, and $B_{4 y z}$ are proportional to the first derivative at zero strain of the third-order polynomials of (e), (f), and (g). 
Direct DFT Calculations

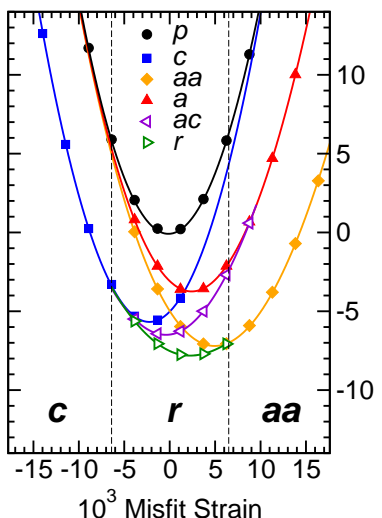

Direct DFT Calculations

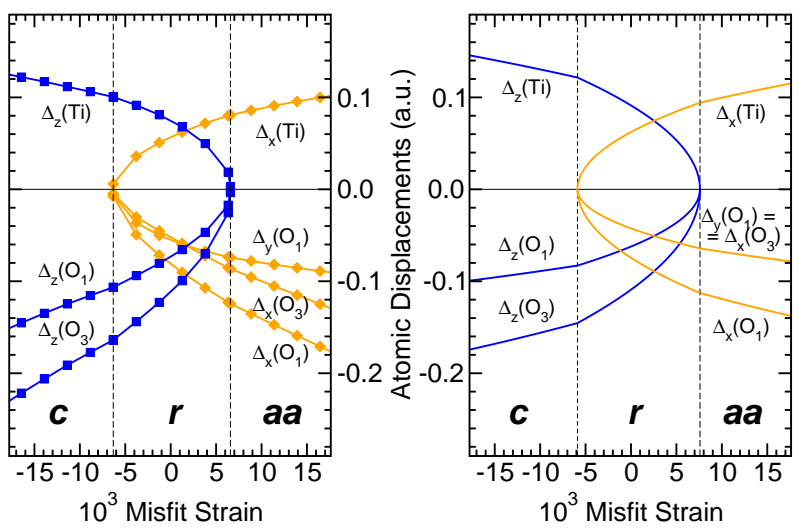

Figure 5: Top panel: comparison of $\mathrm{BaTiO}_{3}$ energy curves for the six epitaxial phases studied, as obtained from direct DFT calculations [9] (left), and from the KSV theory (right). Energies are relative to the paraelectric structure at zero misfit strain. The lines in the left panel and the symbols in the right panel are provided as guides to the eye. Bottom panel: Comparison of $\mathrm{BaTiO}_{3}$ atomic displacements for the most stable phase at each given value of strain, as obtained from direct DFT calculations 9 (left), and from our KSV model (right). $\Delta_{z}(\mathrm{Ti})$ indicates the displacement of the Ti atom along the $z$ direction, etc. Symmetry implies that $\Delta_{y}(\mathrm{Ti})=\Delta_{x}(\mathrm{Ti}), \Delta_{x}\left(\mathrm{O}_{2}\right)=\Delta_{y}\left(\mathrm{O}_{1}\right), \Delta_{y}\left(\mathrm{O}_{2}\right)=\Delta_{x}\left(\mathrm{O}_{1}\right), \Delta_{z}\left(\mathrm{O}_{2}\right)=\Delta_{z}\left(\mathrm{O}_{1}\right)$, and $\Delta_{y}\left(\mathrm{O}_{3}\right)=\Delta_{x}\left(\mathrm{O}_{3}\right)$. The lines in the left panel are guides to the eye. 


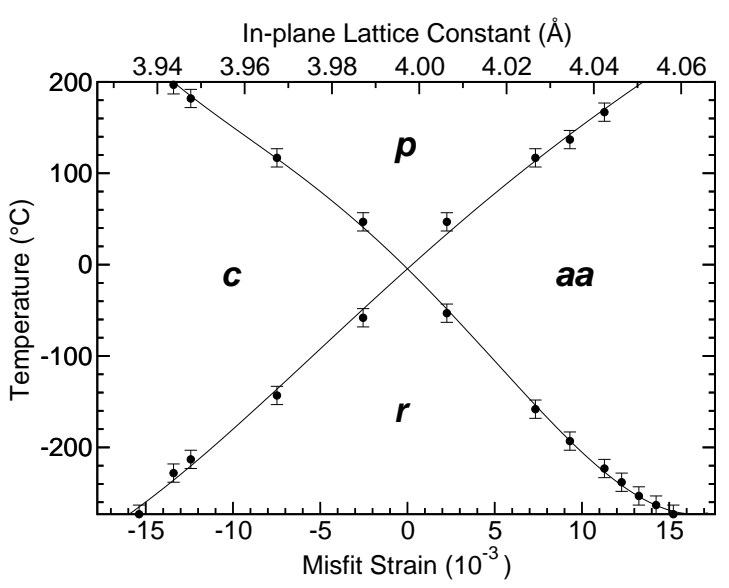

Figure 6: Phase diagram of epitaxial $\mathrm{BaTiO}_{3}$ obtained using the effective Hamiltonian of Zhong, Vanderbilt and Rabe 29, 30. All transitions are second-order.

\subsection{Finite-temperature calculations}

The calculations presented so far were all done at zero temperature. In Ref. [9], we extended our study of epitaxial $\mathrm{BaTiO}_{3}$ to finite temperatures by using the effective Hamiltonian approach of Zhong, Vanderbilt, and Rabe. 29, 30, This method follows the spirit of the first-principles-based calculations described above, but now the model is expanded to allow for different distortions in each individual unit cell. The relevant degrees of freedom are taken to consist of a ferroelectric local-mode vector in each cell; a displacement vector in each cell; and the global homogeneous strain variables. The ferroelectric local-mode and the displacement variables describe local (in general inhomogeneous) polarizations and strains, respectively. The number of parameters needed to describe such a model is larger, since one needs to include new coefficients that quantify the interactions between local-mode variables on neighboring sites. However, all of the coefficients in the expanded model are again obtained from fitting to an appropriately constructed database of first-principles results. Finally, the effective-Hamiltonian model obtained in this way can be used as the basis for finite-temperature Monte Carlo (MC) simulations that can be used to map out finite-temperature phase diagrams.

It is straightforward to impose the constraint of fixed in-plane strain by fixing three of the six elements of the homogeneous strain tensor during the MC simulations. For each value of in-plane strain, MC thermal averages are obtained for the unconstrained components of the homogeneous strain and the average polarization, and phase transitions are identified by monitoring the symmetries of these quantities. The resulting Pertsev diagram is shown in Fig. [6] it is reproduced from Ref. 9], where additional technical details were given.

The Pertsev diagrams of Figs. 1(a), 1(b), and 6 share the same topology above and just below $T_{\mathrm{C}}$ : $p$ at high temperature, $c$ at large compressive misfit, $a a$ at large tensile misfit, and a four-phase point connecting these phases with the $r$ phase at $T_{\mathrm{C}}$. At lower temperature, there is a drastic difference between Figs. 1(a) and 1(b), with our theory supporting the latter. While our theory underestimates the temperature of the 4-phase crossing point in Fig. 6 by about $100{ }^{\circ} \mathrm{C}$, this is the price we pay for insisting on a first-principles approach; indeed, this effective Hamiltonian underestimates the temperature of the bulk cubic-to-tetragonal transition by about the same amount.

\section{Studies of other perovskite films}

In this section we review work on the effect of misfit strain on perovskite films other than $\mathrm{BaTiO}_{3}$ using first-principles methodologies similar to the ones described in the previous section. 


\section{1 $\quad \mathrm{KNbO}_{3}$}

$\mathrm{KNbO}_{3}$ is a perovskite whose bulk form is very similar to that of $\mathrm{BaTiO}_{3}$. It displays the same sequence of transitions with decreasing temperature: cubic to tetragonal to orthorhombic to rhombohedral, with five-atom unit cells.

Using our zero-temperature first-principles-base approach, we found 10 that $\mathrm{KNbO}_{3}$ and $\mathrm{BaTiO}_{3}$ also behave in a very similar way in terms of their behavior as a function of epitaxial strain in the context of film geometries. $\mathrm{KNbO}_{3}$ shows the same $c \rightarrow r \rightarrow a a$ sequence of transitions as does $\mathrm{BaTiO}_{3}$ when varying the misfit strain at zero temperature in the absence of external perpendicular stress. The first transition occurs at a misfit strain of $-4.8 \times 10^{-3}$, and the second occurs at $5.5 \times 10^{-3}$.

\section{$4.2 \quad \mathrm{PbTiO}_{3}$}

As in the cases of $\mathrm{BaTiO}_{3}$ and $\mathrm{KNbO}_{3}$, bulk $\mathrm{PbTiO}_{3}$ has a ferroelectric ground state with a five-atom unit cell. In this case, however, there is only one transition, from cubic to tetragonal, as the temperature is reduced.

A very similar approach to the full first-principles one presented in Sec. 3.1 was used by Bungaro and Rabe to study the influence of misfit strain on $\mathrm{PbTiO}_{3}$ films 31. As in the case of $\mathrm{BaTiO}_{3}$, they find a $c \rightarrow r \rightarrow a a$ sequence of phase transitions, although in this case the range of stability of the $r$ phase is narrower than for $\mathrm{BaTiO}_{3}$ (from $+6.6 \times 10^{-3}$ to $+14.8 \times 10^{-3}$ ), and occurs only at expansive strains. In the same paper, these authors analyze a superlattice with alternating layers of $\mathrm{PbTiO}_{3}$ and $\mathrm{PbZrO}_{3}$, finding again the $c \rightarrow r \rightarrow a a$ sequence of transitions, but with a wider region of $r$ behavior than in the pure $\mathrm{PbTiO}_{3}$ case.

Our first-principles-based approach of Ref. [10] also predicts that $\mathrm{PbTiO}_{3}$ adopts the $c$ phase for compressive strains and the $a a$ phase for tensile strains, with a window of around $1 \%$ misfit strain in between (from $-3.0 \times 10^{-3}$ to $8.4 \times 10^{-3}$ ). However, we find that the $r$ phase is less energetically favorable than a combination of $c$ and $a a$ domains.

\section{3 $\quad \mathrm{SrTiO}_{3}$}

This perovskite is a "quantum paraelectric" in bulk form, meaning that it is only the zero-point motion of ions that prevents ferroelectricity from developing. The cubic phase undergoes a non-ferroelectric oxygen-tilting (or antiferrodistortive) transition at about $105 \mathrm{~K}$.

Antons and coworkers 32] carried out a full first-principles investigation along the lines we have described in Sec. 3.1 for $\mathrm{SrTiO}_{3}$ films with five atoms in the unit cell. They found that in this case the sequence of second-order transitions is $c \rightarrow p \rightarrow a a$ as the strain goes from compressive to tensile. The paraelectric phase exists for misfit strains between $-7.5 \times 10^{-3}$ and $+5.4 \times 10^{-3}$. They also observed a strong dependence of the dielectric properties of these films on the misfit strain. A similar behavior was observed by us in [10, although with a narrower window of $p$ phase in between the $c$ and $a a$ phases.

In their first-principles study, Lin, Huang, and Guo 33. considered larger unit cells for $\mathrm{SrTiO}_{3}$, including in this way the possibility of having antiferrodistortive instabilities in the films. As in the previous studies, they find that the polarization goes from pointing in the [001] direction for sufficiently compressive strains to pointing in the [110] direction for sufficiently tensile strains. The paraelectric window extends in their case from $-4 \times 10^{-3}$ to $+4 \times 10^{-3}$. Their phase diagram is richer due to the presence of the new phases that involve rotations of the oxygen octahedra.

\section{4 $\mathrm{KTaO}_{3}$}

As in the case of $\mathrm{SrTiO}_{3}$, ferroelectricity is suppressed in bulk $\mathrm{KTaO}_{3}$ by quantum fluctuations. In Ref. 34. Akbarzadeh and coworkers use a first-principles based method to draw the Pertsev diagram for $\mathrm{KTaO}_{3}$ films. Unlike the theories described above, this work includes electrostatic boundary conditions and finite-size effects, albeit in an approximate way. For films that are $28 \AA$ thick, these authors find that quantum fluctuations have almost no effect when the electrodes are ideal (short-circuit boundary conditions). In this case, the diagram is very similar to that of strong ferroelectrics like $\mathrm{BaTiO}_{3}$. When they simulate more realistic electrodes, they find that the corresponding depolarizing fields in the film 
strongly affect the phase diagram. Under these conditions, the quantum fluctuations might affect the phase diagram and eventually suppress the $r$ phase.

\subsection{Other perovskites}

Bulk $\mathrm{CaTiO}_{3}$ experiences a phase transition from the cubic phase (with a five-atom unit cell) to an orthorhombic phase (with a twenty-atom unit cell) as temperature is reduced. Our calculations [10] for $\mathrm{CaTiO}_{3}$ films show a similar behavior as for the $\mathrm{PbTiO}_{3}$ ones, with the window between $c$ and $a a$ phases (misfit strains of $-2.3 \times 10^{-3}$ to $5.35 \times 10^{-3}$ ) showing a mixture of $c$ and $a a$ domains.

The ground state of bulk $\mathrm{NaNbO}_{3}$ shows a ferroelectric monoclinic phase with twenty atoms in the unit cell. The behavior of the film form [10] is similar to that of $\mathrm{KNbO}_{3}$, with a $c \rightarrow r \rightarrow a a$ sequence of transitions occurring at misfit strains of $5.5 \times 10^{-3}$ and $4.1 \times 10^{-3}$.

Bulk $\mathrm{PbZrO}_{3}$ undergoes a transition from cubic to an antiferroelectric phase that has forty atoms in the unit cell. In Ref. [10] we show that the five-atom unit cell film stays in the $r$ phase except for large misfit strains that are experimentally difficult to achieve. The behavior of the true ground-state structure with epitaxial strain has yet to be fully explored.

$\mathrm{BaZrO}_{3}$ maintains the simple cubic structure in bulk form at all temperatures. According to our previous study [10], it also remains paraelectric in film form for all experimentally relevant misfit strains,

\section{Summary}

In this article we have reviewed the role that first-principles calculations have played in understanding the effects of substrate-imposed misfit strain on epitaxially grown perovskite ferroelectric films. To do so we have analyzed the case of $\mathrm{BaTiO}_{3}$, complementing our previous publications on this subject with unpublished data to help explain in detail how these calculations are done. In particular, we have added new explanations and figures to clarify how we carried out a stability analysis to identify precisely the critical misfit strains at which phase transitions occur, how we checked for the possible effects of octahedral rotations, and how we computed the coefficients of our first-principles based Landau-Devonshire theory. We have also reviewed similar studies in the literature for other perovskite ferroelectric-film materials.

The use of first-principles calculations to understand the effects of misfit strain on the properties of films is not restricted to the type of materials discussed here. For example, Fennie and Rabe [35] have suggested that misfit strain can be used as a mechanism to obtain strong coupling between ferroelectric and magnetic ordering in $\mathrm{EuTiO}_{3}$. Even more recently, Ishida and Liebsch [36. have found that, when grown epitaxially on $\mathrm{SrTiO}_{3}, \mathrm{LaTiO}_{3}$ becomes a highly correlated metal, instead of being a Mott insulator as found in the bulk form.

We have shown that important insights can be gained by isolating the effects of misfit strain on the properties of ferroelectric films. In the future, it would be desirable to extend the theory by developing similar systematic approaches capable of handling other effects that are relevant for films, including electric boundary conditions, domain formation, surface and interface effects, and the role of vacancies and impurities.

This work was supported in part by ONR Grant No. N00014-05-1-0054.

\section{References}

[1] C. H. Ahn, K. M. Rabe, and J.-M. Triscone, Science 303, 488 (2004).

[2] M. Dawber, K. M. Rabe, and J. F. Scott, Rev. Mod. Phys. 77, 1083 (2005).

[3] J. Junquera and P. Ghosez, in Handbook of Theoretical and Computational Nanotechnology, edited by M. Rieth and W. Schommers, American Scientific, Stevenson Ranch CA (2006).

[4] I. A. Kornev, H. Fu, and L. Bellaiche, J. Mater. Sci. 41, 137 (2006). 
[5] A.-B. Posadas, M. Lippmaa, F. J. Walker, M. Dawber, C. H. Ahn, and J.-M. Triscone, Top. Appl. Phys. 108, 219 (2007).

[6] K. M. Rabe, Curr. Opin. Solid St. Mater. Sci. 9, 122 (2005).

[7] J. Schwarzkopf and R. Fornari, Prog. Cryst. Growth. Ch. 52, 159 (2006).

[8] J. F. Scott, Science 315, 954 (2007).

[9] O. Diéguez, S. Tinte, A. Antons, C. Bungaro, J. B. Neaton, K. M. Rabe, and D. Vanderbilt, Phys. Rev. B 69, 212101 (2004).

[10] O. Diéguez, K. M. Rabe, and D. Vanderbilt, Phys. Rev. B 72, 144101 (2005).

[11] N. A. Pertsev, A. G. Zembilgotov, and A. K. Tagantsev, Phys. Rev. Lett. 80, 1988 (1998).

[12] N. A. Pertsev, A. G. Zembilgotov, and A. K. Tagantsev, Ferroelectrics 223, 79 (1999).

[13] P. Hohenberg and W. Kohn, Phys. Rev. 136, B864 (1964).

[14] W. Kohn and L. J. Sham, Phys. Rev. 140, A1133 (1965).

[15] X. Gonze J.-M. Beuken, R. Caracas, F. Detraux, M. Fuchs, G.-M. Rignanese, L. Sindic, M. Verstraete, G. Zerah, F. Jollet, M. Torrent, A. Roy, M. Mikami, Ph. Ghosez, J.-Y. Raty, and D. C. Allan, Comput. Mater. Sci. 25, 478 (2002). Visit www. abinit.org.

[16] S. Baroni, A. Dal Corso, S. de Gironcoli, P. Giannozzi, C. Cavazzoni, G. Ballabio, S. Scandolo, G. Chiarotti, P. Focher, A. Pasquarello, K. Laasonen, A. Trave, R. Car, N. Marzari, A. Kokalj, www. quantum-espresso.org.

[17] iJ. M. Soler, E. Artacho, J. D. Gale, A. García, J. Junquera, P. Ordejón, and D. Sánchez-Portal, J. Phys.: Condens. Matter 14, 2745 (2002). Visit www.uam.es/siesta.

[18] G. Kresse and J. Hafner, Phys. Rev. B 47, 558 (1993); G. Kresse and J. Furthmüller, Phys. Rev. B 54, 11169 (1996).

[19] P. E. Blöchl, Phys. Rev. B 50, 17953 (1994).

[20] D. M. Ceperley and B. J. Alder, Phys. Rev. Lett. 45, 566 (1980).

[21] D. J. Singh, Ferroelectrics 194, 299 (1997).

[22] H. J. Monkhorst and J. D. Pack, Phys. Rev. B 13, 5188 (1976).

[23] K. Capelle, Braz. J. Phys. 36, 1318 (2006).

[24] Y. L. Li, S. Choudhury, Z. K. Liu and L. Q. Chen, Appl. Phys. Lett. 83, 1608 (2003).

[25] R. D. King-Smith and D. Vanderbilt, Phys. Rev. B 49, 5828 (1994).

[26] J. S. Speck and W. Pompe, J. Appl. Phys. 76, 766 (1994).

[27] N. A. Pertsev and V. G. Koukhar, Phys. Rev. Lett. 84, 3722 (2000).

[28] D. Vanderbilt, Phys. Rev. B 41, 7892 (1990).

[29] W. Zhong, R. D. King-Smith, and D. Vanderbilt, Phys. Rev. Lett. 72, 3618 (1994).

[30] W. Zhong, D. Vanderbilt, and K. M. Rabe, Phys. Rev. B 52, 6301 (1995).

[31] C. Bungaro and K. M. Rabe, Phys. Rev. B 69, 184101 (2004).

[32] A. Antons, J. B. Neaton, K. M. Rabe, and D. Vanderbilt, Phys. Rev. B 71, 024102 (2005).

[33] C.-H. Lin, C.M. Huang, and G. Y. Guo, J. Appl. Phys. 100, 084104 (2006). 
[34] A. R. Akbarzadeh, L. Bellaiche, J. Íñiguez, and D. Vanderbilt, Appl. Phys. Lett. 90, 242918 (2007).

[35] C. Fennie and K. M. Rabe, Phys. Rev. Lett. 97, 267602 (2006).

[36] H. Ishida and A. Liebsh, arXiv:0711.0516 (2007). 